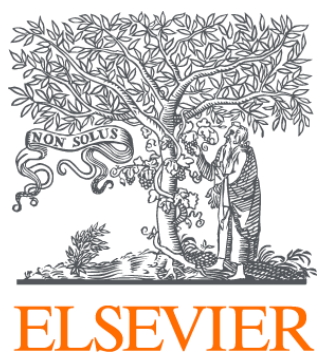

Since January 2020 Elsevier has created a COVID-19 resource centre with free information in English and Mandarin on the novel coronavirus COVID-

19. The COVID-19 resource centre is hosted on Elsevier Connect, the company's public news and information website.

Elsevier hereby grants permission to make all its COVID-19-related research that is available on the COVID-19 resource centre - including this research content - immediately available in PubMed Central and other publicly funded repositories, such as the WHO COVID database with rights for unrestricted research re-use and analyses in any form or by any means with acknowledgement of the original source. These permissions are granted for free by Elsevier for as long as the COVID-19 resource centre remains active. 


\title{
Negative impact of hyperglycaemia on tocilizumab therapy in Covid-19 patients
}

\author{
R. Marfella ${ }^{\mathrm{a}, 1}$, P. Paolisso ${ }^{\mathrm{b}, 1}$, C. Sardu ${ }^{\mathrm{a}, \mathrm{c}, *}$, L. Bergamaschi $^{\mathrm{b}}$, E.C. D’Angelo ${ }^{\mathrm{b}}$, M. Barbieri ${ }^{\mathrm{a}}$,

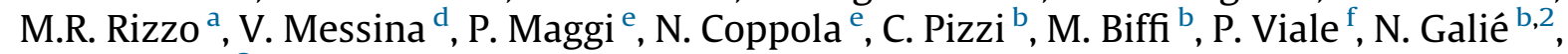 \\ G. Paolisso ${ }^{a, 2}$
}

a Department of Advanced Medical and Surgical Sciences (DAMSS), University of Campania 'Luigi Vanvitelli', 80138 Naples, Italy
b Unit of Cardiology, Department of Experimental, Diagnostic and Specialty Medicine-DIMES, University of Bologna, Italy
${ }^{\mathrm{c}}$ Department of Medical Sciences, International University of Health and Medical Sciences 'Saint Camillus', Rome, Italy
${ }^{\mathrm{d}}$ Department of Infectious Diseases, Sant'Anna Hospital, Caserta, Italy
${ }^{\mathrm{e}}$ Department of Mental and Physical Health and Preventive Medicine, University of Campania 'Luigi Vanvitelli', 80138 Naples, Italy
${ }^{\mathrm{f}}$ Unit of Infectious Diseases, Department of Medical and Surgical Sciences, S. Orsola Hospital, University of Bologna, Italy

\section{A R T I C L E I N F O}

\section{Article history:}

Received 4 May 2020

Received in revised form 13 May 2020

Accepted 14 May 2020

Available online 21 May 2020

\section{Keywords:}

Covid-19

Diabetes mellitus

Interleukin-6

\begin{abstract}
A B S T R A C T
Tocilizumab (TCZ) is used for treating moderate-to-severe Covid-19 pneumonia by targeting interleukin-6 receptors (IL-6Rs) and reducing cytokine release. Yet, in spite of this therapy, patients with vs. patients without diabetes have an adverse disease course. In fact, glucose homoeostasis has influenced the outcomes of diabetes patients with infectious diseases. Of the 475 Covid-19-positive patients admitted to infectious disease departments (University of Bologna, University Vanvitelli of Napoli, San Sebastiano Caserta Hospital) in Italy since 1 March 2020, 31 (39.7\%) hyperglycaemic and 47 (60.3\%) normoglycaemic patients (blood glucose levels $\geq 140 \mathrm{mg} / \mathrm{dL}$ ) were retrospectively evaluated at admission and during their hospital stay. Of note, 20 (64\%) hyperglycaemic and 11 (23.4\%) normoglycaemic patients had diabetes $(P<0.01)$. At admission, hyperglycaemic vs. normoglycaemic patients had fivefold higher IL-6 levels, which persisted even after TCZ administration $(P<0.05)$. Intriguingly, in a risk-adjusted Cox regression analysis, TCZ in hyperglycaemic patients failed to attenuate risk of severe outcomes as it did in normoglycaemic patients $(P<0.009)$. Also, in hyperglycaemic patients, higher IL-6 plasma levels reduced the effects of TCZ, while adding IL-6 levels to the Cox regression model led to loss of significance $(P<0.07)$ of its effects. Moreover, there was evidence that optimal Covid-19 infection management with TCZ is not achieved during hyperglycaemia in both diabetic and non-diabetic patients. These data may be of interest to currently ongoing clinical trials of TCZ effects in Covid-19 patients and of optimal control of glycaemia in this patient subset.
\end{abstract} Crown Copyright (c) 2020 Published by Elsevier Masson SAS. All rights reserved.
To calm the inflammatory storm, tocilizumab (TCZ) has been used in the treatment of moderate-to-severe Covid-19 pneumonia by targeting interleukin-6 receptors (IL-6Rs) and reducing cytokine release [1]. Yet, despite the optimal management of Covid-19 infection with treatments including TCZ, the pooled rate ratio for diabetes patients with adverse disease courses vs. those with more favourable courses was 2.26 [2].

An important factor in any infection outcomes in patients with diabetes may be glucose control [2]. Indeed, hyperglycaemia is

\footnotetext{
* Corresponding author at: Professor of Internal Medicine, DAMSS, University of Campania 'Luigi Vanvitelli', Piazza Miraglia 2, 80138 Napoli, Italy.

E-mail address: drsarducele@gmail.com (C. Sardu).

1 Co-first authors.

2 Co-last authors.
}

associated with higher levels of IL-6 and IL-6Rs [3], both of which are predictors of severe lung disease in Covid-19 patients [1]. However, at this time, there are no data on the effects of TCZ on outcomes in hyperglycaemic Covid-19 patients with moderate-to-severe respiratory illness. To investigate this unresolved need, 475 Covid-19-positive patients, admitted to infectious disease departments (University of Bologna, University Vanvitelli Napoli, San Sebastiano Caserta Hospital) in Italy since 1 March 2020, were retrospectively studied. The study protocol was approved by the Ethics Committees of the relevant Institutional Review Boards.

The diagnosis of Covid-19 was established according to World Health Organization (WHO) interim guidance and confirmed by RNA detection of severe acute respiratory syndrome coronavirus 2 (SARS-CoV-2) in the hospitals' microbiology laboratories. Of the 
consecutive patients receiving TCZ therapy for moderate-to-severe respiratory illness due to Covid-19 pneumonia, only 78 were further evaluated because of severe outcomes, encompassing both the use of mechanical ventilation and death. The remainder of the study population, which failed to meet this inclusion criteria, was thereafter excluded from the study analysis.

TCZ was administered to all patients as an intravenous (IV) infusion in doses of $8 \mathrm{mg} / \mathrm{kg}$, using a prefilled syringe, up to a maximum of $800 \mathrm{mg}$ per dose, with an additional dose $8-12 \mathrm{~h}$ later if clinically required. A total of 31 (39.7\%) hyperglycaemic and 47 (60.3\%) normoglycaemic Covid-19-positive patients (blood glucose levels $\geq 140 \mathrm{mg} / \mathrm{dL}$ at admission and during their hospital stay) were evaluated [3]. Of these, 20 (64\%) hyperglycaemic and 11 (23.4\%) normoglycaemic patients had diabetes $(P<0.01$; Table $S 1$; see supplementary material associated with this article online). Diabetes was diagnosed according to the evidence of fasting plasma glucose $(\mathrm{PG}) \geq 7.0 \mathrm{mmol} / \mathrm{L}(126 \mathrm{mg} / \mathrm{dL})$ or $2-\mathrm{h} P G \geq 11.1 \mathrm{mmol} / \mathrm{L}$ $(200 \mathrm{mg} / \mathrm{dL})$ and by a patient history of known diabetes and use of antidiabetic drugs [4]. Admission glucose levels were $187 \pm 48 \mathrm{mg} /$ dL vs. $103 \pm 23 \mathrm{mg} / \mathrm{dL}(P<0.01)$ in hyperglycaemic vs. normoglycaemic patients, respectively. During hospitalization, mean glucose levels were $157 \pm 15 \mathrm{mg} / \mathrm{dL}$ vs. $122 \pm 12 \mathrm{mg} / \mathrm{dL}(P<0.01)$ in hyperglycaemic vs. normoglycaemic patients, respectively.

In the study population, there were no differences in the treatment used to control hyperglycaemia, as all patients admitted to hospital were receiving insulin therapy while all hypoglycaemic drug treatments were stopped. In addition, subcutaneous insulin was begun at the time of admission in all hyperglycaemic patients with or without a previous diabetes diagnosis: short-acting insulin was given before meals; and intermediate or long-acting insulin in the evening. Patients with previous diabetes diagnoses at admission stopped taking oral antidiabetic drugs, including metformin, sulphonylureas, dipeptidyl peptidase (DPP)-IV inhibitors, sodium-glucose cotransporter-2 (SGLT2) inhibitors and glucagon-like peptide (GLP)-1 receptor agonists.
At admission, higher IL-6 levels were found in hyperglycaemic patients that persisted even after TCZ administration (Fig. 1A). Also, in the risk-adjusted Cox regression analysis, TCZ in hyperglycaemic patients failed to attenuate the risk of severe outcomes as it did in normoglycaemic patients $(P<0.009$; Fig. 1B). Moreover, Kaplan-Meier analysis showed that hyperglycaemic patients without a diabetes diagnosis had an increased risk of severe disease compared with both normoglycaemic and hyperglycaemic patients with a previous diabetes diagnosis (Fig. S1; see supplementary material associated with this article online).

These data are in agreement with previous observations by Capes et al. [5], who found that patients without diabetes, but with stress hyperglycaemia on admission for acute myocardial infarction, were at greater risk of in-hospital mortality than patients previously diagnosed with diabetes. Several possible mechanisms may explain this observation. Patients with diabetes are more likely to receive insulin and/or oral antidiabetic drugs for hyperglycaemia before and during acute illness [5], and such treatments may reduce the rise in free radicals and inflammation, and also decrease coagulability because of a reduced production of plasminogen activator inhibitor type-1 (PAI-1) activity and proinsulin-like molecules [6]. On the other hand, higher IL-6 serum levels may blunt the effects of TCZ in hyperglycaemic patients as per numerous observations.

Initially, severe Covid-19 patients might experience cytokine release syndrome (CRS), a negative prognostic factor for survival in such patients $[1,2,7,8]$. CRS damps down type- 1 interferon (IFN) responses in airway and alveolar epithelial cells, thereby allowing rapid viral replication while also attracting an excessive number of inflammatory monocytes/macrophages, neutrophils, dendritic cells and natural-killer (NK) cells into the lungs [7]. As a consequence, the result could be an overactivation of innate responses through a cytokine-driven vicious cycle [8]. In addition, pulmonary injury might be assisted by diffuse alveolar damage, dysregulated coagulation and pulmonary fibrinolysis due to
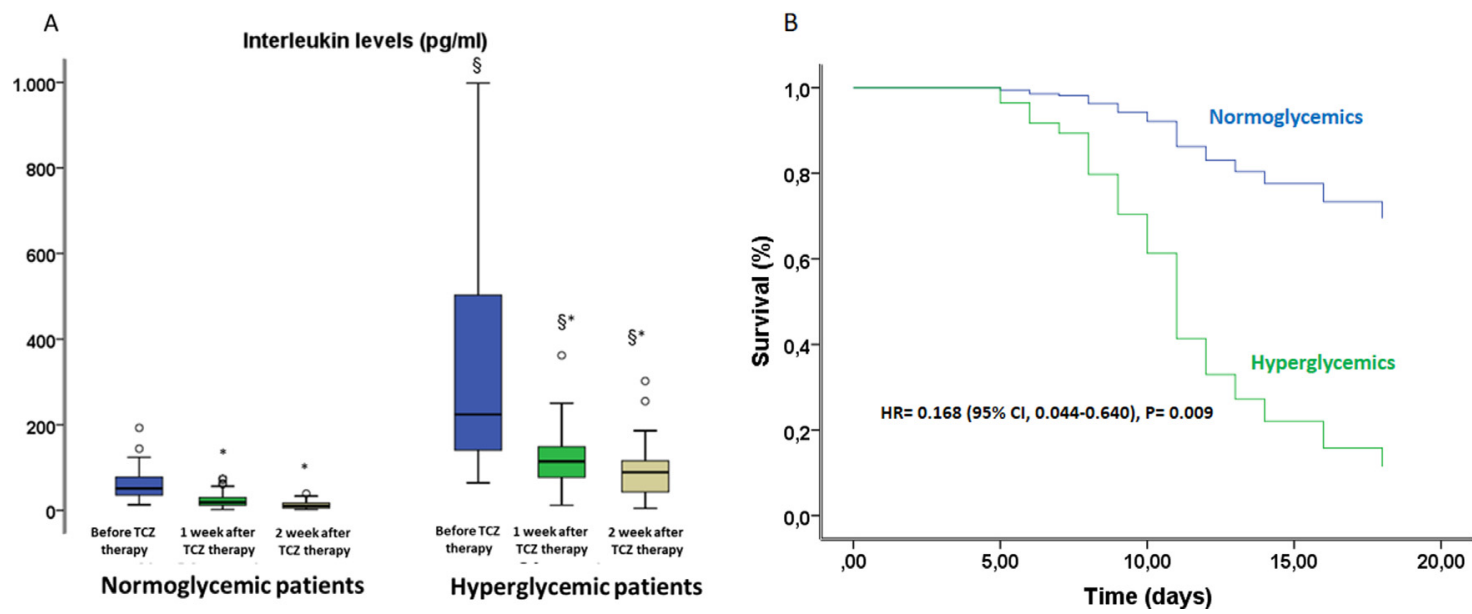

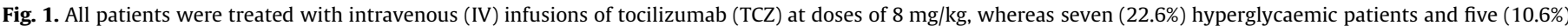

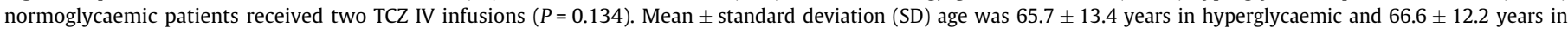

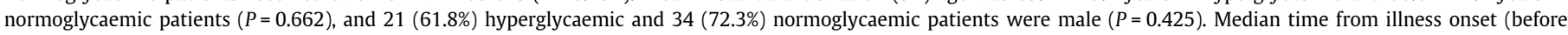

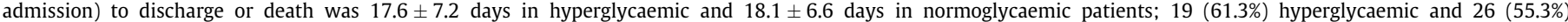

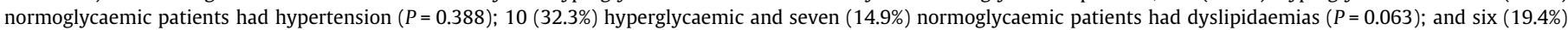

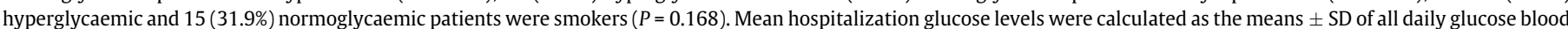

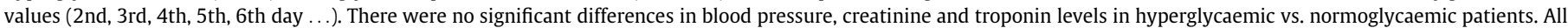

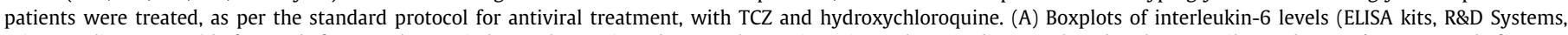

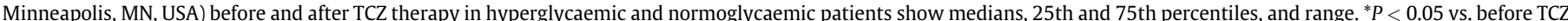

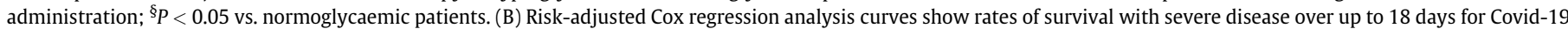

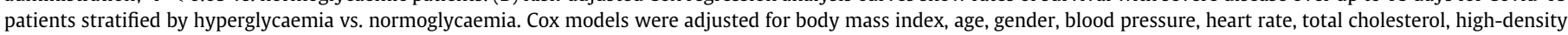

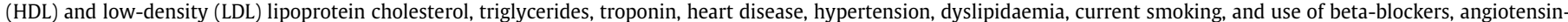

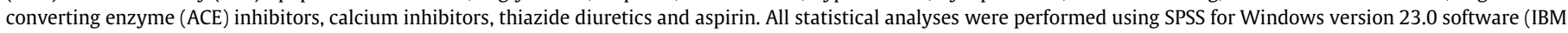
Corp., Armonk, NY, USA). Two-sided $P<0.05$ was considered statistically significant. HR: hazard ratio. 
the overwhelming presence of proinflammatory cytokines and chemokines [8]. Furthermore, CRS could leak into the systemic circulation to cause extrapulmonary manifestations and a multiple organ dysfunction syndrome (MODS) [8].

In addition, it may be that hyperglycaemia impacts different components of the host response, including immune cell function and cytokine regulation [3]. Interestingly, serum concentrations of proinflammatory cytokines, including IL-2 receptors (IL-2Rs), IL-6 and tumour necrosis factor (TNF)- $\alpha$, were increased in the majority of severe cases and were markedly higher compared with moderate cases, suggesting that cytokine storms could be associated with disease severity [8]. Similarly, SARS has also been characterized by exuberant inflammatory responses and lung damage [8]. A previous study, using a mouse model of SARS, demonstrated that the rapid kinetics of SARS-CoV replication and a delay in type-1 IFN signalling promoted inflammatory monocyte/ macrophage accumulation, resulting in elevated lung cytokine/ chemokine levels, vascular leakage and suboptimal T-cell responses [8].

In the present study, hyperglycaemic patients presented with higher IL-6 levels compared with normoglycaemic patients. Moreover, at admission, higher PG levels were associated with higher plasma IL-6 levels and, despite full therapy for Covid-19 infection, hyperglycaemic patients presented with higher levels of IL-6 than normoglycaemic patients during hospitalization. Thus, elevated blood glucose may itself trigger an inflammatory response leading to severe Covid-19 disease and death.

Intriguingly, IL-6 is one of the key cytokines in CRS, with a central role in acute inflammation, autoimmune cell differentiation and human metabolism $[7,8]$. In addition, IL-6 promotes T-cell population expansion/activation and B-cell differentiation, and also regulates lipid metabolism and insulin resistance leading to systemic organ damage [8]. In this setting, TCZ through its targeting of the IL- 6 pathways is a blocker of the IL- 6 signal transduction pathway and can be used in patients with severe Covid-19 [8]. Notably, based on a previous study conducted in patients with rheumatoid arthritis, TCZ efficacy was shown to be inversely proportional to baseline IL-6 levels [9]. Moreover, in Covid-19 patients, TCZ treatment led to a significant decline in inflammatory markers, with radiological improvement and reduced ventilator support requirements [10]. Ongoing clinical trials are currently evaluating the safety/efficacy effects of TCZ according to screening and monitoring parameters [11].

The present study has demonstrated that hyperglycaemic patients had fivefold higher IL-6 levels than normoglycaemic ones at the time of hospital admission. Interestingly, when IL-6 levels were added to the Cox regression model, the significance of the TCZ effect was lost $(P<0.07)$. Therefore, hyperglycaemia might be seen as a metabolic marker of IL-6 overproduction [3], and could be a precondition of baseline overexpression of IL-6, as reflected by TCZ effectiveness and the poorer prognosis of severe Covid-19 patients. However, the lack of glycosylated haemoglobin $\left(\mathrm{HbA}_{1 \mathrm{c}}\right)$ values for our study population according to patients with vs. without type 2 diabetes (T2D) could be a limiting factor, as it does not allow any conclusions to be drawn concerning hyperglycaemia causes and effects in patients without T2D. Moreover, hyperglycaemia may be a reflection of either unknown (but still present) diabetes or a marker of more severe infection in a stressful condition (such as respiratory distress or hypoxia).

When taken together, our present observations indicate that optimal Covid-19 infection management with TCZ could be not achieved during hyperglycaemia in both diabetic and non-diabetic patients. Thus, these data may be of interest to ongoing clinical trials of TCZ effects in Covid-19 patients and the optimal control of glycaemia in this patient subset.

\section{Authors' contributions}

Study design and conception: Raffaele Marfella, Pasquale Paolisso, Celestino Sardu, Giuseppe Paolisso.

Data collection: Luca Bergamaschi, Emanuela Concetta D’Angelo, Michelangela Barbieri, Maria Rosaria Rizzo.

Data analysis: Raffaele Marfella.

Study revision: Vincenzo Messina, Paolo Maggi, Nicola Coppola, Carmine Pizzi, Mauro Biffi, PierluigiViale, Nazzareno Galié.

\section{Disclosure of interest}

The authors declare that they have no competing interest.

\section{Appendix A. Supplementary data}

Supplementary data associated with this article can be found, in the online version, at https://doi.org/10.1016/j.diabet.2020.05.005.

\section{References}

[1] Zhang C, Wu Z, Li JW, Zhao H, Wang GQ. The cytokine release syndrome (CRS) of severe COVID-19 and Interleukin-6 receptor (IL-6R) antagonist Tocilizumab may be the key to reduce the mortality. Int J Antimicrob Agents 2020. http:// dx.doi.org/10.1016/j.ijantimicag.

[2] Maddaloni E, Buzzetti R. Covid-19 and diabetes mellitus: unveiling the interaction of two pandemics. Diabetes Metab Res Rev 2020;e33213321. http:// dx.doi.org/10.1002/dmrr.3321.

[3] Esposito K, Nappo F, Marfella R, Giugliano F, Ciotola M, Quagliaro L, et al. Inflammatory cytokine concentrations are acutely increased by hyperglycemia in humans: role of oxidative stress. Circulation 2002;106:2067-72.

[4] Classification and Diagnosis of Diabetes. American Diabetes Association. Diabetes Care 2015;38(Suppl. 1):S8-16.

[5] Capes SE, Hunt D, Malmberg K, Gerstein HC. Stress hyperglycaemia and increased risk of death after myocardial infarction in patients with and without diabetes: a systematic overview. Lancet 2000;355:773-8.

[6] Jain SK, Nagi DK, Slavin BM, Lumb PJ, Yudkin JS. Insulin therapyin type 2 diabetic patients suppresses plasminogen activator inhibitor(PAI-1) activity and proinsulin-like molecules independently of glycaemic control. Diabetes Med 1993;10:27-32.

[7] Bingwen L, Min L, Zhiguang Z, Xuan G, Yufei X. Can we use interleukin-6 (IL-6) blockade for coronavirus disease 2019 (COVID-19)-induced cytokine release syndrome (CRS)? J Autoimmunity 2020. http://dx.doi.org/10.1016/j.jaut. 2020.102452

[8] Chi Zhang, Zhao Wu, Jia-Wen Li, Hong Zhao, Gui-Qiang Wang. The cytokine release syndrome (CRS) of severe COVID-19 and Interleukin-6 receptor (IL-6R) antagonist Tocilizumab may be the key to reduce the mortality. International J Antimicr Agents 2020. http://dx.doi.org/10.1016/j.ijantimicag.2020.105954.

[9] Aizu M, Mizushima I, Nakazaki S, Nakashima A, Kato T, Murayama T, et al. Changes in serum interleukin-6 levels as possible predictor of efficacy of tocilizumab treatment in rheumatoid arthritis. Mod Rheumatol 2018; 28:592-8.

[10] Alattar R, Ibrahim TBH, Shaar SH, Abdalla S, Shukri K, Daghfal JN, et al. Tocilizumab for the treatment of severe coronavirus disease 2019. J Med Virol 2020. http://dx.doi.org/10.1002/jmv.25964 [Epub ahead of print].

[11] Alzghari SK, Acuña VS. Supportive treatment with tocilizumb in COVID-19: a systematic review. J Clin Virol 2020;127:104380. http://dx.doi.org/10.1016/ j.jcv.2020.104380 [Epub ahead of print]. 